\section{OPEN ACCESS}

Edited by:

Guillermina Hernandez-Raquet, Institut National de la Recherche

Agronomique (INRA), France

Reviewed by:

Michael J. Rothrock,

Agricultural Research Service (USDA),

United States

Edward Pajarillo,

Florida A\&M University, United States

*Correspondence:

Zhuocheng Hou

zchou@cau.edu.cn

Ning Yang

nyang@cau.edu.cn

${ }^{t}$ These authors have contributed equally to this work

Specialty section:

This article was submitted to Systems Microbiology, a section of the journal

Frontiers in Microbiology

Received: 23 December 2018

Accepted: 29 August 2019

Published: 13 September 2019

Citation:

Yan W, Sun C, Zheng J, Wen C,

Ji C, Zhang D, Chen Y, Hou Z and

Yang N (2019) Efficacy of Fecal Sampling as a Gut Proxy in the Study

of Chicken Gut Microbiota.

Front. Microbiol. 10:2126.

doi: 10.3389/fmicb.2019.02126

\title{
Efficacy of Fecal Sampling as a Gut Proxy in the Study of Chicken Gut Microbiota
}

Wei Yan ${ }^{1 \dagger}$, Congjiao Sun ${ }^{1 \dagger}$, Jiangxia Zheng ${ }^{1}$, Chaoliang Wen ${ }^{1}$, Congliang $\mathrm{Ji}^{2}$,
Dexiang Zhang ${ }^{2}$, Yonghua Chen ${ }^{2}$, Zhuocheng Hou ${ }^{1 *}$ and Ning Yang ${ }^{1 *}$

' National Engineering Laboratory for Animal Breeding and MOA Key Laboratory of Animal Genetics and Breeding, College of Animal Science and Technology, China Agricultural University, Beijing, China, ${ }^{2}$ Wen's Nanfang Poultry Breeding Co. Ltd., Yunfu, China

Despite the convenience and non-invasiveness of fecal sampling, the fecal microbiota does not fully represent that of the gastrointestinal (Gl) tract, and the efficacy of fecal sampling to accurately represent the gut microbiota in birds is poorly understood. In this study, we aim to identify the efficacy of feces as a gut proxy in birds using chickens as a model. We collected 1,026 samples from 206 chickens, including duodenum, jejunum, ileum, cecum, and feces samples, for $16 \mathrm{~S}$ rRNA amplicon sequencing analyses. In this study, the efficacy of feces as a gut proxy was partitioned to microbial community membership and community structure. Most taxa in the small intestine (84.11-87.28\%) and ceca (99.39\%) could be identified in feces. Microbial community membership was reflected with a gut anatomic feature, but community structure was not. Excluding shared microbes, the small intestine and ceca contributed 34.12 and $5.83 \%$ of the total fecal members, respectively. The composition of Firmicutes members in the small intestine and that of Actinobacteria, Bacteroidetes, Firmicutes, and Proteobacteria members in the ceca could be well mirrored by the observations in fecal samples $(\rho=0.54-0.71$ and 0.71-0.78, respectively, $P<0.001)$. However, there were few significant correlations for each genus between feces and each of the four gut segments, and these correlations were not high $(\rho=-0.2-0.4, P<0.05)$ for most genera. Our results suggest that fecal microbial community has a good potential to identify most taxa in the chicken gut and could moderately mirror the microbial structure in the intestine at the microbial population level with phylum specificity. However, it should be interpreted with caution by using feces as a proxy to study associations for microbial structure at individual microorganism level.

Keywords: gut microbiota, feces, proxy, spatial relationships, chicken

\section{INTRODUCTION}

Many studies have reported the important roles of gut microbiota in host metabolism and health in humans (Rosenbaum et al., 2015), other mammals (Shin et al., 2014), and birds (Brisbin et al., 2008). Because of the convenience and non-invasiveness of fecal sampling, most studies use fecal samples as a proxy to study the gut microbiota, despite the increasing recognition that fecal 
microbial populations may not be fully representative of those in the contents or mucosa of the gastrointestinal (GI) tract (Gevers et al., 2014; Lavelle et al., 2015). Therefore, a comprehensive understanding of the efficacy of using fecal samples as a proxy to study the GI microbiota would help improve longitudinal analyses of microbiota and the application of fecal samples (McCormack et al., 2017; Johnson et al., 2018).

Among birds, the chicken is frequently used as a research model, and its GI microbiota has been studied previously (Dumonceaux et al., 2006; Yeoman et al., 2012; Stanley et al., 2014; Shaufi et al., 2015; Clavijo and Mjv, 2018). In several studies, the microbiota present in different GI segments have been investigated using traditional sequencing methods (Gong et al., 2007) or high-throughput sequencing techniques (Choi et al., 2014; Xiao et al., 2016). However, these studies had small sample sizes $(N=3-8)$, were primarily aimed at examining the spatial heterogeneity among different segments, and did not focus on the spatial microbiota relationships between feces and the GI tract.

Compared with most mammals, the cecum in birds has been reported to play important roles in metabolism, such as in the digestion of cellulose, starch, and other resistant polysaccharides (Mead, 1989; Clench and Mathias, 1995) and in the absorption of nutrients (Obst and Diamond, 1989) and water (Gasaway et al., 1976). Microbial compositions and functions in chicken ceca have been reported in many studies (Stanley et al., 2013; Sergeant et al., 2014). In addition, Stanley et al. (2015) examined the microbial relationships between the ceca and feces and observed that $88.55 \%$ of all operational taxonomic units (OTUs) were shared. However, the microbial relationships between the ceca and small intestine (including the duodenum, jejunum, and ileum), which would help provide an integrated view of gut microbial relationships, were rarely reported.

Here, we performed large-scale sequencing surveys and focused on the efficacy of using feces to represent the GI microbiota in chickens. The efficacy was partitioned into microbial community membership and structure to gain a comprehensive view to improve our understanding of the efficacy of the use of feces as a proxy to study the gut microbiota and their spatial relationships in the gut.

\section{MATERIALS AND METHODS}

\section{Animal Model}

The complete procedure was performed according to the guidelines established by the Animal Care and Use Committee of China Agricultural University (permit number: SYXK 2015-0028).

The pure line (N204) slow-growing yellow broiler, which has been selected for feather color and body conformation for 10 years, was used as the animal model in this study. The birds were obtained from Wen's Nanfang Poultry Breeding Co., Ltd. in Guangdong Province of China. Two hundred and six birds with similar body weights were selected and raised on the ground with ad libitum feeding and nipple drinkers. The birds were fed a common maize-soybean-based diet throughout the duration of the experiment (see Supplementary Table S1 for detailed ingredients information). No antibiotics were applied during the experimental period.

\section{Sample Collection}

Fresh fecal samples were collected from each bird as soon as excreta was discharged through the cloaca at 77 days of age with the average body weight $2.32 \mathrm{~kg}$. Next, all the birds were humanely euthanized by cervical dislocation and subsequently dissected. The contents and mucosal surfaces of the duodenum, jejunum, ileum, and cecum were collected immediately after dissection. To ensure the consistency of samples among individuals, a 10-cm-long fixed section of the duodenum and jejunum, the whole ileum, and a pair of ceca were selected for sampling from each bird. The two ends of the selected section of duodenum, jejunum, and ileum were hold and locked by forceps. The selected intestinal section was cut off and the forceps at the end closer to cloaca were removed to let the content get into the storage tube with appropriate squeezing. Next, the intestinal section was opened and the mucosa was scraped from the end closer to proventriculus to the end closer cloaca. The contents and mucosa were mixed uniformly before storage. Since ceca are blind-ended and the content in ceca is stickier than that in the small intestine, we cut off ceca and collected samples as above described without using forceps. All samples were immediately placed in liquid nitrogen and then stored at $-80^{\circ} \mathrm{C}$. Both the intestinal contents and mucosa were sampled based on the consideration that the microbes from both sources may contribute to host interactions with respect to nutrient metabolism and immunity (Smith et al., 2015).

\section{DNA Extraction and 16S rRNA Gene Sequencing}

DNA was extracted from intestinal and fecal samples using a QIAamp DNA stool mini kit (QIAGEN, cat\#51504) (Zhao et al., 2013) following the manufacturer's instructions. PCR amplification of the V4 region of the bacterial 16S rRNA gene was performed using the forward primer $520 \mathrm{~F}\left(5^{\prime}\right.$ AYTGGGYDTAAAGNG-3 $3^{\prime}$ ) and the reverse primer $802 \mathrm{R}\left(5^{\prime}-\right.$ TACNVGGGTATCTAATCC-3'). Sample-specific 7-bp barcodes were incorporated into the primers for multiplex sequencing. The PCR reactions contained $5 \mu \mathrm{l}$ of Q5 reaction buffer $(5 \times), 5 \mu l$ of Q5 High-Fidelity GC buffer $(5 \times), 0.25 \mu l$ of Q5 High-Fidelity DNA Polymerase (5 U/ $\mu \mathrm{l}), 2 \mu \mathrm{l}$ of dNTPs $(2.5 \mathrm{mM}), 1 \mu \mathrm{l}(10 \mu \mathrm{M})$ of each forward and reverse primer, $2 \mu \mathrm{l}$ of DNA template, and $8.75 \mu \mathrm{l}$ of $\mathrm{ddH}_{2} \mathrm{O}$. Thermal cycling consisted of initial denaturation at $98^{\circ} \mathrm{C}$ for $2 \mathrm{~min}$, followed by 25 cycles of denaturation at $98^{\circ} \mathrm{C}$ for $15 \mathrm{~s}$, annealing at $55^{\circ} \mathrm{C}$ for $30 \mathrm{~s}$, and extension at $72^{\circ} \mathrm{C}$ for $30 \mathrm{~s}$, with a final extension at $72^{\circ} \mathrm{C}$ for 5 min. PCR amplicons were purified using Agencourt AMPure Beads (Beckman Coulter, Indianapolis, IN, United States) and quantified using a PicoGreen dsDNA Assay kit (Invitrogen, 
Carlsbad, CA, United States). After the quantification step, amplicons were pooled in equal amounts, and $2 \times 300 \mathrm{bp}$ paired-end sequencing was performed using an Illumina MiSeq platform with the MiSeq Reagent kit v3 at Shanghai Personal Biotechnology Co., Ltd. (Shanghai, China). The raw data on which the conclusions of the manuscript rely have been deposited in the National Center for Biotechnology Information (NCBI) database (accession numbers SRP139192, SRP139193, and SRP139195).

\section{Analysis of Sequencing Data}

Data analysis was performed using the Quantitative Insights Into Microbial Ecology (QIIME, v1.8.0) pipeline (Caporaso et al., 2010), due to the advantages of QIIME (Nilakanta et al., 2014; Plummer and Twin, 2015; Pollock et al., 2018). Briefly, raw sequencing reads with exact matches to the barcodes were assigned to respective samples and identified as valid sequences. The low-quality sequences were filtered based on the following criteria (Gill et al., 2006; Chen and Jiang, 2014): length $<150 \mathrm{bp}$, average Phred score $<20$, ambiguous bases, and mononucleotide repeats $>8$ bp. Pairedend reads were assembled using FLASH (Magoč and Salzberg, 2011), and chimera detection was performed with usearch61 in QIIME. After quality control, four fecal samples were excluded due to low sequence quality that was potentially caused by a technical artifact. The remaining high-quality sequences were clustered into OTUs at a $97 \%$ sequence identity using an open-reference OTU picking protocol against the Silva database (SILVA128) (Quast et al., 2013; Yilmaz et al., 2014; Balvočiūtè and Huson, 2017).

We focused on open-reference OTU picking for these analyses because this method yields substantially more taxonomic identifications with sequences that failed to hit the reference database than do closed-reference methods (Rideout et al., 2014). The open-reference method can provide more information for comparisons among intestinal segments or feces. The singleton OTUs were discarded because such OTUs can occur due to sequencing errors. Only OTUs representing $>0.001 \%$ of the total filtered OTUs were retained to improve the efficiency of the analysis. Because the sequencing and sampling quantity varied among individuals, we rarefied the data to the lowest number of sequences per sample in each site to control for sampling effort in diversity analyses. The sequence cutoff values for duodenum, jejunum, ileum, cecum, and feces were 22,321, 27,709, 24,885, 37,137, and 25,821 , respectively. Beta diversity (weighted and unweighted UniFrac distance) of individual OTUs was calculated with postrarefaction data and the phylogenetic tree. Principal coordinate analysis (PCoA) was performed using the unweighted or weighted UniFrac distance (Lozupone and Knight, 2005) for different intestinal segments and feces. To decrease the data noise, only OTUs that were present in more than three samples at each sampling site were used to analyze the effect of microbial membership. The correlations between the mean fecal and segmental genera abundance were calculated using the method described in a study of rhesus macaques (Yasuda et al., 2015). These methods could primarily provide the number and diversity of microbes in feces and each segment which would help to quantitatively understand the relationships of microbial communities between feces and GI tract.

\section{Statistical Analysis}

Venn plots were generated for intestinal segment or feces samples at the OTU level using the VennDiagram package in $R$ (v3.4.3). PERMANOVA was performed between two of the sampling sites using package vegan in $R$ (v3.5.2). Spearman correlation analysis was performed in package psych in $R$ (v3.4.3). Paired Student's $t$-test was used to compare the microbial UniFrac distance between two sampling sites. Mann-Whitney test was performed to identify the differences of each genus between two sampling sites.

\section{RESULTS}

\section{Sequencing Data}

The 16S rRNA gene-based sequencing from 206 chickens produced 62,193,309 reads, 58,959,487 of which remained after quality filtration. The average number of sequences per sample was 57,465 and the number of sequences per sample ranged from 22,321 to 224,188 .

\section{Landscape and Quantification of Microbial Relationships Among Feces, Ceca, and Small Intestine}

To gain an overview of the microbial relationships among the chicken duodenum, jejunum, ileum, ceca, and feces, unweighted UniFrac distances (community membership; presence/absence of taxa) and weighted UniFrac distances (community structure; taking the relative abundances of taxa into account) were used to perform principal coordinates analysis (PCoA; Figures 1A,B). The variation in community memberships among different sites was primarily explained by the sites origin (Figure 1A), but the community structures showed both the sites origin and interindividual variation (Figure 1B). In particular, the cecal microbial community exhibited a distant relationship with the small intestine community, and the microbial community in feces showed an intermediate relationship between those of the ceca and small intestines.

UniFrac distances between two samples from all assayed sites within each individual were calculated to quantify the spatial relationships of the gut microbiota. When the community membership was considered alone, the UniFrac distance decreased along the gut anatomical locations from the farthest to the nearest sites between fecal and duodenal, jejunal, ileal, or cecal samples (FD, FJ, FI, or FC, respectively, in Figure 1C), presenting clear anatomical differences. However, when taking the community structure into account, the UniFrac distance increased in FI and FC compared with that in FJ (Figure 1D). This finding might be explained by the exchange of contents between the 


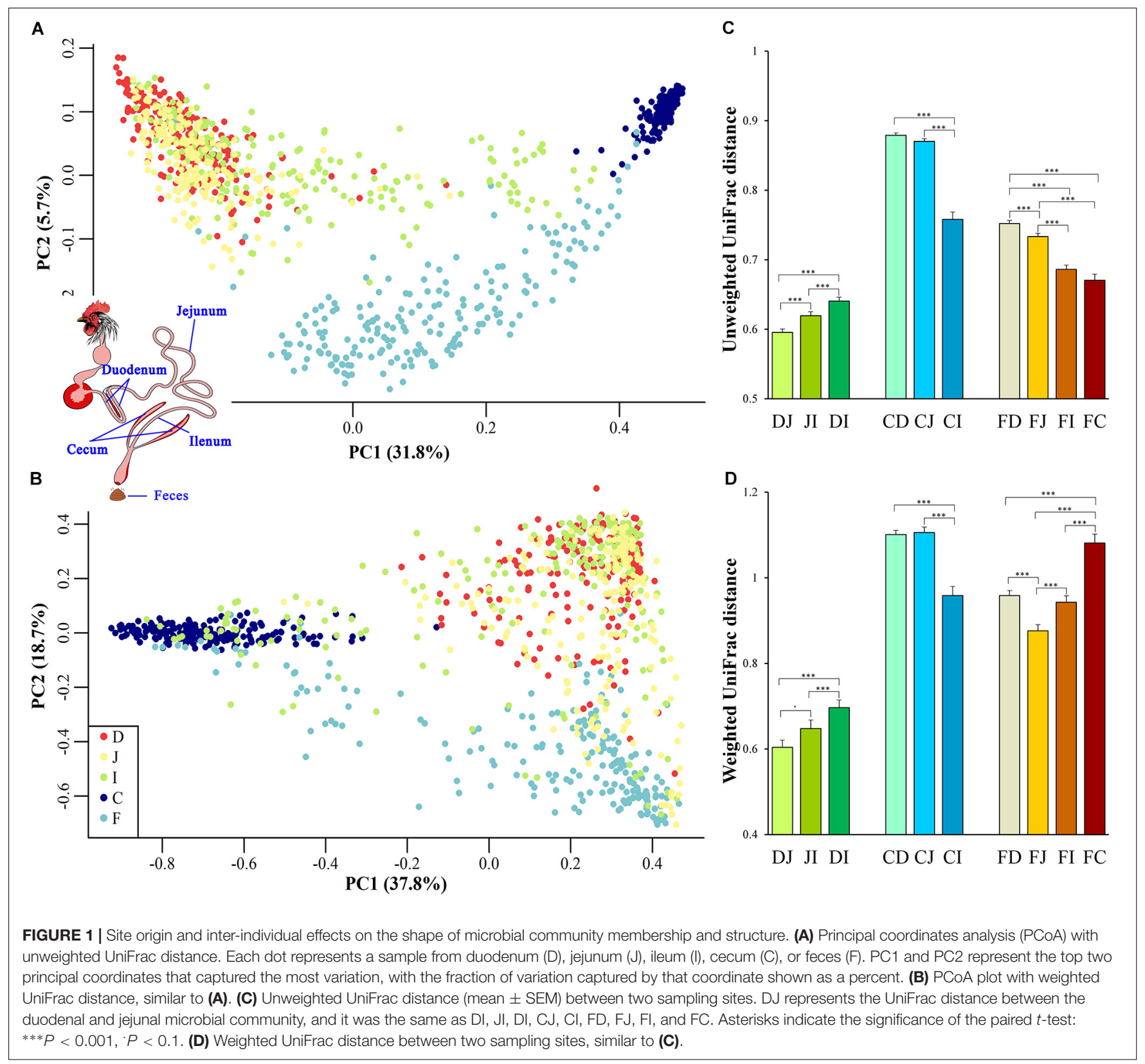

ileum and ceca, suggesting that the specific cecal microbial structure influences the microbial communities in the ileum and feces.

Among all pairs, the unweighted UniFrac distance between the cecal and duodenal as well as jejunum samples was highest $(P<0.05)$, and that between duodenal and jejunal samples was lowest $(P<0.05$; Figure 1C and Supplementary Tables S2, S3). Regarding the weighted UniFrac distances, cecal samples had similar distances to the duodenal and jejunal samples, and these distances were greater than for the other pairs $(P<0.05)$, whereas the lowest distance was observed between duodenal and jejunal samples $(P<0.1$; Figure 1D and Supplementary Tables S2, S3). These results suggest that limited differences exist within small intestinal microbial communities, while the microbial structure in the ceca is quite distinct from those in the small intestine. The above results were also supported by the PERMANOVA analysis as shown in Table 1.

\section{Analyses of Shared and Exclusive Microbial Members}

Given that both community membership and structure influence the microbial relationships among the feces, ceca, and small intestine, we next evaluated the extent to which the spatial relationships were influenced by the above two factors. The shared and exclusive OTUs were calculated to assess the influence of the microbial community membership. To decrease the data noise, only OTUs present in more than three samples at each sampling site were used to analyze the effect of 
TABLE 1 | PERMANOVA analysis for microbiota in different sampling sites.

\begin{tabular}{ll}
\hline Sampling sites & $\boldsymbol{R}^{\mathbf{2}(\mathbf{2})}$ \\
\hline D-J(1) & 0.02 \\
D-I & 0.07 \\
J-I & 0.05 \\
C-D & 0.58 \\
C-J & 0.56 \\
C-I & 0.44 \\
F-D & 0.39 \\
F-J & 0.35 \\
F-I & 0.28 \\
F-C & 0.39 \\
\hline
\end{tabular}

(1) $D, J, I, C$, and $F$ denote duodenum, jejunum, ileum, cecum, and feces, respectively. ${ }^{(2)} R^{2}$ denotes the proportions of variances that could be explained by the grouping.

microbial membership. We observed that 971 OTUs, accounting for $30.9 \%$ of the total OTUs, were shared across all sites (Figure 2A), and these shared OTUs can be referred to as the "core" microbiota in the gut. These OTUs represented different proportions of sequences in different sites and were especially high in fecal samples (96.50\%; Figure 2B), indicating that the most abundant members detected in fecal samples belonged to these "core" microbiota. At the genus level, these core taxa were primarily classified as Bacteroides, Intestinibacter, Lactobacillus, Rikenellaceae RC9 gut group, and Gallibacterium (Supplementary Figure S1A). It is noteworthy that $5.88 \%$ of the "core" microbiota sequences were not assigned and that most of these sequences $(71.40 \%)$ were detected in the cecal samples (small pie chart in Supplementary Figure S1A), suggesting that most of these unassigned taxa tended to be anaerobic microbes.

Most OTUs in the small intestine (84.11-87.28\%) and cecal (99.39\%) samples could be identified as fecal OTUs (Table 2), indicating that feces would be a good proxy for identifying species in the gut microbiota. However, some OTUs that were present in the GI tract $(12.72-15.89 \%$ in small intestinal and $0.61 \%$ in cecal samples) remained undetected in fecal samples (Table 2) and members of Clostridiales, Rhizobiales, Xanthomonadales, and Bacteroidales appeared to be particularly undetected in feces (Supplementary Table S4).

Microbial communities in the small intestine and ceca did not contribute equally to the fecal microbial members, as $35.18 \%$ of fecal OTUs were not identified in cecal samples, most of which $(34.12 \%)$ could be identified in small intestinal niches (Table 2 and Figure 2C). These OTUs were primarily from the orders Clostridiales, Lactobacillales, Pseudomonadales, Rickettsiales, and so on (Supplementary Figure S1B) and were considered
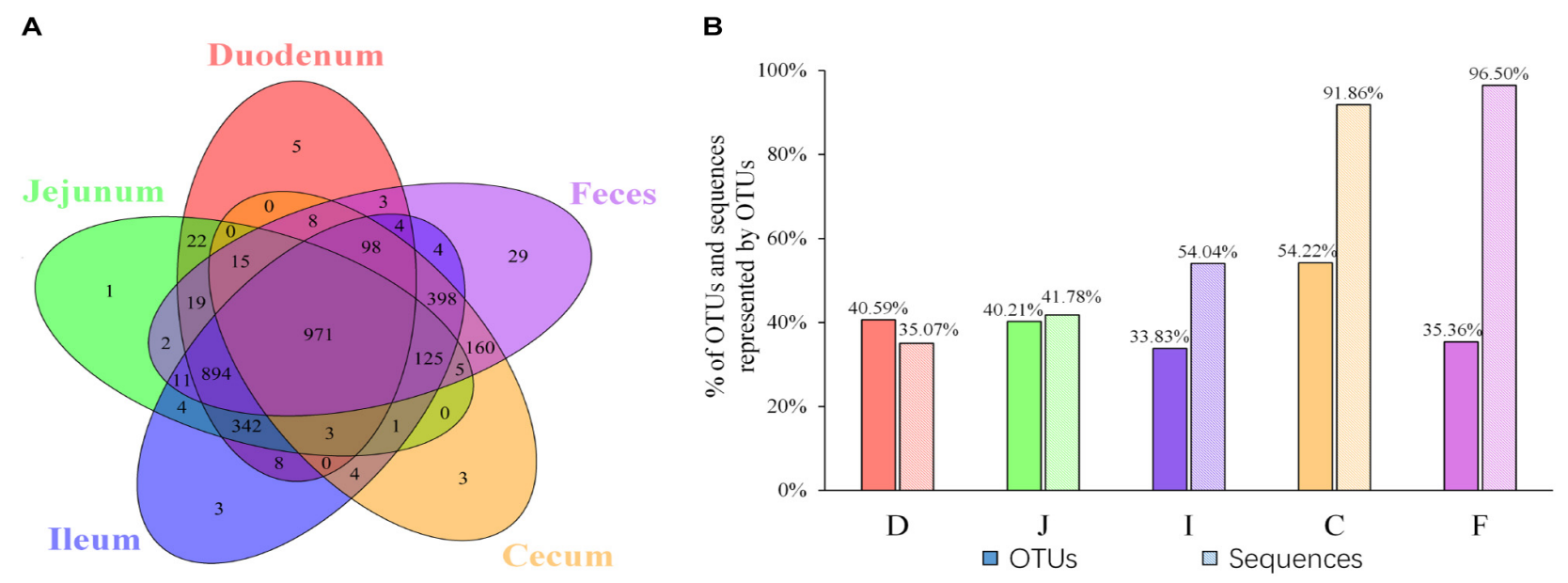

C

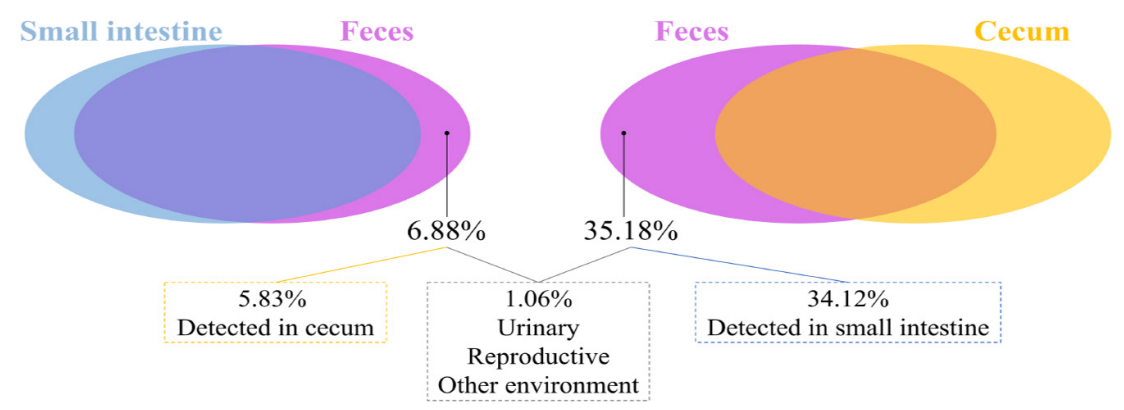

FIGURE 2 | OTUs shared across different sampling sites. (A) Venn diagram demonstrating that the taxa overlap among different sampling sites. (B) The percentage of core OTUs and sequences represented by these OTUs in the duodenal (D), jejunal (J), ileal (I), cecal (C), and fecal (F) samples. (C) The percentage of OTUs in feces exclusively contributed by small intestine or cecum, and the percentage of OTUs in feces was below the limit of detection in the gastrointestinal tract. 
TABLE 2 | Shared and exclusive OTUs between two of sampling sites.

\begin{tabular}{|c|c|c|c|c|c|}
\hline \multirow[t]{2}{*}{ Site1 } & \multirow[t]{2}{*}{ Site2 } & \multicolumn{2}{|c|}{ Shared OTUs } & \multicolumn{2}{|c|}{ Exclusive OTUs } \\
\hline & & In site1 (\%) & In site2 (\%) & In site1 (\%) & In site2 (\%) \\
\hline \multirow[t]{2}{*}{$D^{1}$} & J & $94.73^{2}$ & 93.83 & 5.27 & 6.17 \\
\hline & & $99.86^{3}$ & 99.94 & 0.14 & 0.06 \\
\hline \multirow[t]{2}{*}{$\mathrm{D}$} & 1 & 96.99 & 80.84 & 3.01 & 19.16 \\
\hline & & 99.57 & 99.28 & 0.43 & 0.72 \\
\hline \multirow[t]{2}{*}{ J } & I & 97.35 & 81.92 & 2.65 & 18.08 \\
\hline & & 99.79 & 99.48 & 0.21 & 0.52 \\
\hline \multirow[t]{2}{*}{ C } & $\mathrm{D}$ & 61.14 & 45.78 & 38.86 & 54.22 \\
\hline & & 92.78 & 35.19 & 7.22 & 64.81 \\
\hline \multirow[t]{2}{*}{ C } & $J$ & 62.53 & 46.38 & 37.47 & 53.62 \\
\hline & & 93.28 & 41.86 & 6.72 & 58.14 \\
\hline \multirow[t]{2}{*}{ C } & I & 89.34 & 55.75 & 10.66 & 44.25 \\
\hline & & 99.17 & 54.75 & 0.83 & 45.25 \\
\hline \multirow[t]{2}{*}{$\mathrm{F}$} & $\mathrm{D}$ & 73.27 & 84.11 & 26.73 & 15.89 \\
\hline & & 98.57 & 96.94 & 1.43 & 3.06 \\
\hline \multirow[t]{2}{*}{$\mathrm{F}$} & J & 74.36 & 84.55 & 25.64 & 15.45 \\
\hline & & 98.69 & 97.84 & 1.31 & 2.16 \\
\hline \multirow[t]{2}{*}{$\mathrm{F}$} & । & 91.22 & 87.28 & 8.78 & 12.72 \\
\hline & & 99.23 & 97.02 & 0.77 & 2.98 \\
\hline \multirow[t]{2}{*}{ F } & C & 64.82 & 99.39 & 35.18 & 0.61 \\
\hline & & 98.13 & 99.96 & 1.87 & 0.04 \\
\hline
\end{tabular}

${ }^{1} D, J, I, C$, and F denotes the microbial community of duodenum, jejunum, ileum, cecum, and feces, respectively. ${ }^{2}$ The percentage of shared or exclusive OTUs.

${ }^{3}$ The percentage of sequences shared or exclusive OTUs represent.

exclusive contributors of the small intestinal microbiota to fecal microbial members. The ceca exclusively contributed $5.83 \%$ of OTUs to the observed fecal members, representing $0.28 \%$ of the fecal sample sequences and consisting of taxa primarily from the orders Bacteroidales, Rhizobiales, Clostridiales, Micrococcales, and Flavobacteriales (Figure 2C and Supplementary Figure S1C).

\section{Correlation Analyses of Microbial Abundances}

Because community structure also affects the spatial relationships of gut microbiota, we next performed Spearman correlation analyses between the mean fecal and segmental genera abundance to evaluate the effects of community structure and assess the extent to which the microbial community in the GI tract was reflected in the fecal samples (Figure 3). If a high correlation was observed between two sites, the differences in abundance between sites were considered highly consistent, so that the abundance at one site had the potential to be a good proxy for the abundance at another. The microbial composition of feces was correlated with those in the small intestine (Spearman: $\rho=0.38 ; P<0.001$ ) and in the combination of small intestine and ceca $(\rho=0.48 ; P<0.001$; Figure 3). We then performed similar analyses to identify the correlation bias in predominant phyla (Actinobacteria, Bacteroidetes, Firmicutes, and Proteobacteria; Supplementary Figure S2). Genera of the Firmicutes and Proteobacteria phyla in fecal samples showed moderate to high correlations with those at all four GI sites $(\rho=0.40-0.76, P<0.001)$. In particular,

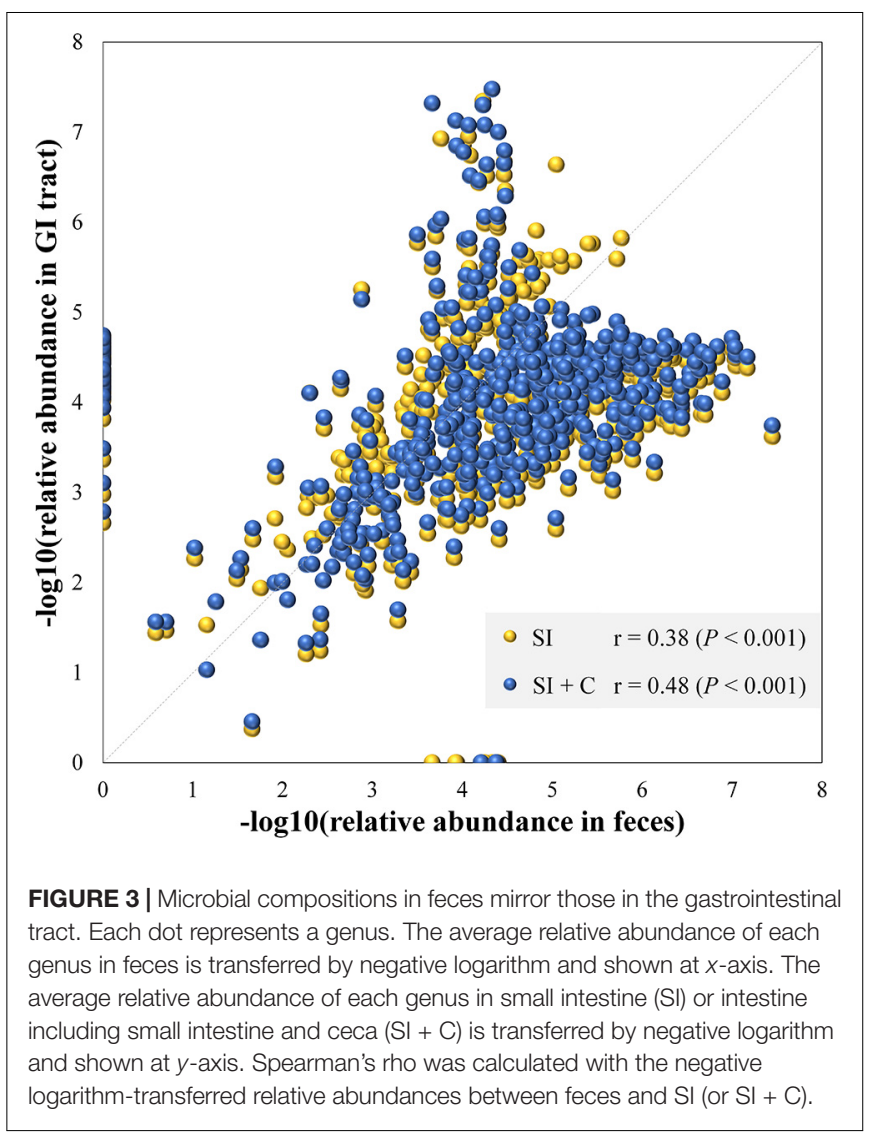

fecal samples were well representative of Firmicutes members in both the small intestine and ceca $(\rho=0.54-0.71, P<0.001)$ and of Actinobacteria, Bacteroidetes, and Proteobacteria members in the ceca $(\rho=0.74-0.78, P<0.001)$. However, Actinobacteria members in the small intestine might not be well represented in fecal samples $(\rho=0.13-0.22, P>0.05)$.

A follow-up question concerned the extent to which each microbe correlated between two sites. To address this issue, Spearman correlation tests were performed for each genus between two sites. The genera with abundances over $0.1 \%$ at either compared site with a significant correlation $(P<0.05)$ are summarized in Figure 4 and Supplementary Table S5. Between the fecal and each of the four gut segmental samples, a limited number of significant correlations $(P<0.05)$ were observed, and these correlations were not high $(\rho=-0.2-0.4, P<0.05)$ for each genus. Most genera with significant correlations belonged to the phyla Firmicutes and Proteobacteria. However, more significant and moderate correlations were observed between two of the small intestinal segments, and most of the genera with significant correlations were also from the phyla Firmicutes and Proteobacteria (Supplementary Table S5). The results suggest that the gut microbiota structures could be moderately reflected by fecal samples when taking all genera into consideration simultaneously, but analyses of fluctuations in abundance for a specific genus should be interpreted with caution.

Although microbes at one site were weakly correlated with the corresponding microbes at another site, certain patterns 


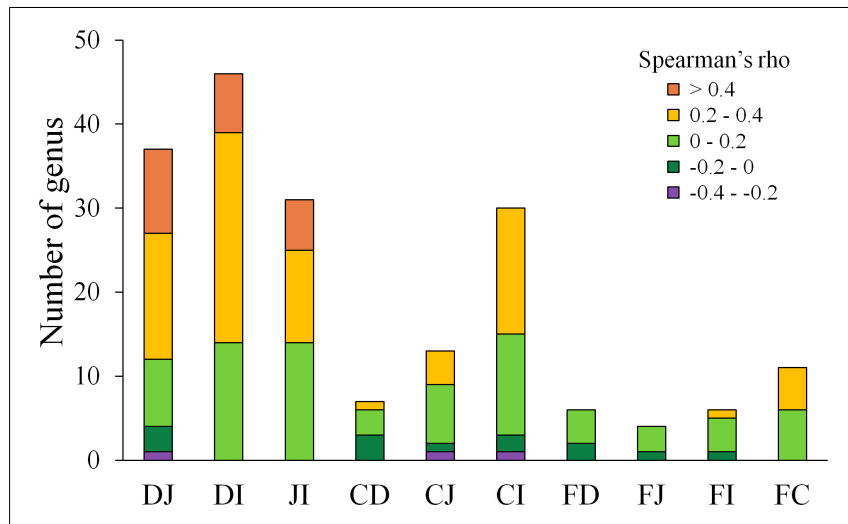

FIGURE 4 | Distribution of Spearman correlations for each genus between two sites. D, J, I, C, and F denote the microbial communities of the duodenum, jejunum, ileum, cecum, and feces, respectively. Only genera with an abundance $>0.1 \%$ at either site of comparison and significant correlations $(P<0.05)$ are shown.

were observed in some cases, as exemplified by the genus Campylobacter (Table 3). The abundance of this genus in ceca exhibited consistent correlations with that observed in the jejunum $(\rho=0.21, P<0.05)$ and ileum $(\rho=0.37, P<0.05)$. In ileal samples, this genus was correlated with that measured in fecal samples $(\rho=0.19, P<0.05)$, while no correlation was observed between cecal and fecal samples. This finding indicates that Campylobacter has great colonization ability in the distal gut of chickens, especially in ceca, and most Campylobacter contributions to the fecal composition are probably from the ileum, but not from the ceca.

\section{DISCUSSION}

This study is a large-scale sequencing assessment of the efficacy of using fecal samples as a proxy for the gut microbiota in birds. In this study, we comprehensively examined the community membership and structure of the chicken gut microbiome at

TABLE 3 | The Spearman correlation for Campylobacter among different sample sites.

\begin{tabular}{lcc}
\hline Sample sites & Spearman correlation & $\boldsymbol{P}$-value \\
\hline D-J & 0.31 & $7.14 \mathrm{E}-06$ \\
D-I & 0.19 & $7.26 \mathrm{E}-03$ \\
D-C & 0.01 & $8.44 \mathrm{E}-01$ \\
J-I & 0.39 & $6.43 \mathrm{E}-09$ \\
J-C & 0.21 & $2.74 \mathrm{E}-03$ \\
I-C & 0.37 & $5.56 \mathrm{E}-08$ \\
F-D & 0.07 & $2.89 \mathrm{E}-01$ \\
F-J & 0.13 & $5.55 \mathrm{E}-02$ \\
F-I & 0.19 & $6.34 \mathrm{E}-03$ \\
F-C & 0.08 & $2.70 \mathrm{E}-01$ \\
\hline
\end{tabular}

${ }^{1} D, \quad J, I, \quad C$, and $F$ denote duodenum, jejunum, ileum, cecum, and feces, respectively. five different biogeographic sites within 206 individual animals. We showed that fecal samples were good proxies for detecting the presence/absence of GI microbial members because most GI tract members could be detected within anatomic features in fecal samples (microbial communities in feces showed increasing similarities to those in the GI tract along the duodenum-jejunum-ileum-ceca axis). However, phyla bias and interindividual effects were observed to affect the efficacy of using fecal samples to study GI microbial abundance.

We also should note that the next-generation sequencing (NGS) approach could not absolutely detect all microbes in the gut because of some limitations of NGS method (Rizzo and Buck, 2012; Daber et al., 2013). Some microbes that may be present at lower levels than the limit of detection. Therefore, some OTUs that were not detected in feces but were found in the small intestine or ceca probably exist but remain below the detection limit or filtration criteria.

Similar to the current study, a high proportion of shared OTUs has been previously observed between fecal and cecal samples in chickens (Stanley et al., 2015). Similarly, a study in house mice observed that $93.3 \%$ of OTUs were shared between fecal and lower GI samples (Suzuki and Nachman, 2016). Another chicken study indicated that the GI origin is a primary determinant for the chicken fecal microbiota composition (Sekelja et al., 2012), supporting the high proportion of shared OTUs between feces and the four gut segments observed in the current study. These results indicate that fecal samples have good potential for identifying microbial members derived from the GI tract. However, another chicken study by Choi et al. (2014) observed low percentages of shared OTUs between segments. A major reason for the differences among studies might be the small sample size in Choi's study, which would increase the sensitivity of the results with respect to individual variation. Moreover, the presence/absence of microbial members in the GI tract was observed to be reflected by fecal samples in a given anatomical feature, i.e., fecal samples had more similarities in community membership to those in ileal and cecal samples than to those in duodenal and jejunal samples, consistent with previous reports in birds (Xiao et al., 2016) and mammals (Gu et al., 2013; Suzuki and Nachman, 2016; Dias et al., 2018).

As for microbial community structure, the efficacy of using fecal samples to represent the gut microbiota structure did not work as well as for community membership. First, the weighted UniFrac distances between feces and each of intestinal segments were significantly higher than the corresponding unweighted UniFrac distances (Supplementary Figure S3), suggesting that taking the abundance into account significantly increased the dissimilarity between feces and each of the GI segments. Second, the abundances of most taxa were significantly different between fecal and GI samples (Supplementary Table S6), consistent with previous studies (Gong et al., 2007; Xiao et al., 2016; Yan et al., 2017). Third, the correlations between the mean fecal and segmental genera abundances were moderate, similar to the results in rhesus macaques (Yasuda et al., 2015). However, these correlations display bias among different phyla, i.e., different phyla in the GI tract are differentially mirrored by fecal samples. Fourth, significant correlations $(P<0.05)$ 
of each microbe between fecal and segmental samples were low and rare, suggesting that the efficacy of using fecal samples to represent microbial abundance was affected by the interindividual effect. A similar effect has also been observed in humans (Lavelle et al., 2015).

Previous studies in humans (Stearns et al., 2011; Lavelle et al., 2015) and other mammals (Yasuda et al., 2015; Li et al., 2017) have also addressed the issue of whether fecal samples are good representatives for GI microbial analyses. Although the conclusions may not be fully consistent, nearly all studies reached a consensus that microbial communities in fecal samples do not represent the whole GI microbiota. Studies in humans suggest that microbial communities in the duodenum and colon are not represented by those in feces because of the large differences in microbial profiles (Stearns et al., 2011), and these studies emphasized the need to examine tissue biopsies in addition to fecal samples (Gevers et al., 2014), proposing that standard forceps mucosal biopsy samples can represent bacterial populations (Lavelle et al., 2015). Compared with human studies, studies in other mammals are more comprehensive because a larger number of gut segments can be involved in the analyses. Several studies in mice (Suzuki and Nachman, 2016; Li et al., 2017) support the utility of fecal samples for studying the gut microbiota, because microbial communities in fecal samples were observed to be similar to those in the lower GI tract, which is supported by studies conducted in rhesus macaques (Yasuda et al., 2015), pigs (Zhao et al., 2015), and equines (Ericsson et al., 2016).

Compared with previous studies, the strength of the current study lies in the following: (1) it involved the use of gut segments from the upper GI tract to the lower GI tract and feces, providing a relatively comprehensive view of the spatial relationships of the gut microbiota; (2) the microbial relationships were partitioned into two parts, i.e., microbial community membership and structure, providing multiangle observations to identify microbial relationships between feces and the GI tract; and (3) a massive number of individuals was sampled, which is significant for investigations of gut spatial relationships, as the sizes of most of the above studies did not exceed 20. The considerable sample size would provide more comprehensive insights into exploring the utility of fecal samples in studies of the gut microbiota.

Because of the specific and significant roles in nutrition and health (Clench and Mathias, 1995; Waite and Taylor, 2014), cecal microbiota have been widely investigated in birds (Matsui et al., 2010; Wienemann et al., 2011), especially chickens (Stanley et al., 2013; Oakley et al., 2014; Sergeant et al., 2014). Bacteroides was observed as the dominant taxa in our study (Supplementary Figure S1D) and in most other studies (Tillman et al., 2011; Wei et al., 2013), although some reports observed a predominance of Clostridiales members in ceca (Cressman et al., 2010; Choi et al., 2014). Although the cecal microbial community may sometimes be linked to diet (Wienemann et al., 2011), the nearly consistent results across studies suggest that the cecal microbial community is stable. This finding might be due to ceca having a special blindended structure and being located in the lower GI tract, providing a stable and anaerobic environment for microbes and longer storage periods of the contents, in contrast to the rapid transit environment in the small intestine (Clench and Mathias, 1992). In addition to the microbial composition, Stanley et al. (2015) also compared microbial differences and similarities between ceca and feces in chicken. They observed that $88.55 \%$ of all OTUs, containing $99.25 \%$ of all sequences, were shared by the ceca and feces, similar to the observations in the current study. These results indicate that except for some rare microbial members, most microbes in the ceca can be detected in fecal samples.

The microbial relationships between the ceca and small intestine have been rarely reported in birds. Choi et al. (2014) compared the percentage of shared OTUs among ceca and three small intestinal sections but observed low percentages between segments (ranging from 1.2 to $2.9 \%$, representing from 38.7 to $65.5 \%$ of sequences). The percentages reported in another study by Xiao et al. (2016) were $60.2 \%$ for the duodenum, $50.5 \%$ for the jejunum, and $43.5 \%$ for the ileum, which were calculated from Figure 3 in their article. In contrast, the results of Xiao's study presented an opposite trend from our findings, i.e., the percentages of shared OTUs in Xiao's study decreased from the duodenum to the jejunum and ileum, demonstrating a reversed-anatomical feature compared with the current study. These inconsistent results might be attributable to differences among species, diets, or other environmental factors, but the small sample size in Xiao's study may be an important reason for these inconsistencies.

The slow-growing yellow broilers were used in this study, as they account for about half share of broiler market in China and the slow-growing broilers are showing their promising values in organic agriculture in some western world, such as European Union (Fanatico et al., 2009). The birds used in this study may not represent all kinds of broiler breeds, including the fastgrowing broiler, but could provide references in some extent. The intensive selections for growth and feed efficiency have made differences for performance quantitatively and qualitatively between slow- and fast-growing broilers (Fanatico et al., 2007a,b, 2008). In the meanwhile, some differences were observed in the GIT between fast-growing broilers and slow-growing (or indigenous) broilers (Schmidt et al., 2009; Mabelebele et al., 2014; Alshamy et al., 2018). However, Mabelebele et al. (2014) also found that the proventriculus, small intestine, and cecum weights were not affected by breed differences, and the similar results were found in the proventriculus, large intestine, and cecum $\mathrm{pH}$ values. Alshamy et al. (2018) observed similar growth curves for GI segments between Ross broilers and dual-purpose chickens. Thus, it seems that the selection for growth rate and feed efficiency have made limited alterations to the chicken GIT. This will facilitate findings in the current study to be a reference to the fast-growing broilers, though further studies with more breeds are also required in the future.

Overall, we assessed the efficacy of using fecal samples to represent GI microbiota in birds and analyzed potential factors affecting this efficacy. With highly shared microbial members, fecal samples have the good potential to be used to detect most microbial species in the small intestine and ceca with gut anatomical features. Regarding to the microbial structure, the microbial community in feces could moderately mirror those 
in the intestine at the microbial population level with phylum specificity. However, the analyses of microbial structures at individual microorganism level using fecal samples as the proxy should be interpreted with caution, as the associations for single one microorganism between feces and each of the four intestinal segments were always low and statistically insignificant. This study attempts to identify the microbial relationships between feces and the intestine in birds, which will help extend our understanding of the bird gut microbiota and provide future directions regarding the usage of fecal samples in the studies of gut microbiome.

\section{ETHICS STATEMENT}

This study was carried out in accordance with the recommendations of the guidelines established by the Animal Care and Use Committee of the China Agricultural University. The protocol was approved by the Animal Care and Use Committee of the China Agricultural University (permit number: SYXK 2015-0028).

\section{AUTHOR CONTRIBUTIONS}

NY and ZH designed the study. WY, CS, JZ, CW, CJ, DZ, and YC collected the samples. WY analyzed the data and wrote the manuscript. CS, $\mathrm{ZH}$, and $\mathrm{NY}$ assisted in data analyzing and

\section{REFERENCES}

Alshamy, Z., Richardson, K. C., Hünigen, H., Hafez, H. M., Plendl, J., and Al Masri, S. (2018). Comparison of the gastrointestinal tract of a dual-purpose to a broiler chicken line: a qualitative and quantitative macroscopic and microscopic study. PLoS One 13:e204921. doi: 10.1371/journal.pone.0204921

Balvočiūtè, M., and Huson, D. H. (2017). SILVA, RDP, greengenes, NCBI and OTT - how do these taxonomies compare? BMC Genomics 18(Suppl. 2):114. doi: 10.1186/s12864-017-3501-4

Brisbin, J. T., Gong, J., and Sharif, S. (2008). Interactions between commensal bacteria and the gut-associated immune system of the chicken. Anim. Health Res. Rev. 9, 101-110. doi: 10.1017/S146625230800145X

Caporaso, J. G., Kuczynski, J., Stombaugh, J., Bittinger, K., Bushman, F. D., Costello, E. K., et al. (2010). QIIME allows analysis of high-throughput community sequencing data. Nat. Methods 7, 335-336.

Chen, H., and Jiang, W. (2014). Application of high-throughput sequencing in understanding human oral microbiome related with health and disease. Front. Microbiol. 5:508. doi: 10.3389/fmicb.2014.00508

Choi, J. H., Kim, G. B., and Cha, C. J. (2014). Spatial heterogeneity and stability of bacterial community in the gastrointestinal tracts of broiler chickens. Poult. Sci. 93, 1942-1950. doi: 10.3382/ps.201403974

Clavijo, V., and Mjv, F. (2018). The gastrointestinal microbiome and its association with the control of pathogens in broiler chicken production: a review. Poult. Sci. 97, 1006-1021. doi: 10.3382/ps/pex359

Clench, M. H., and Mathias, J. R. (1992). A complex avian intestinal motility response to fasting. Am. J. Physiol. 262, 498-504.

Clench, M. H., and Mathias, J. R. (1995). The avian cecum: a review. Wilson Bull. 107, 93-121.

Cressman, M. D., Yu, Z., Nelson, M. C., Moeller, S. J., Lilburn, M. S., and Zerby, H. N. (2010). Interrelations between the microbiotas in the litter and in the intestines of commercial broiler chickens. Appl. Environ. Microbiol. 76, 6572-6582. doi: 10.1128/AEM.00180-10 contributed to the revisions. All authors read and approved the final manuscript.

\section{FUNDING}

The current study was funded in part by National Natural Science Foundation of China (31930105), Programs for Changjiang Scholars and Innovative Research in University (IRT_15R62), and China Agriculture Research System (CARS-40).

\section{ACKNOWLEDGMENTS}

We thank Prof. Guiyun Xu for demonstration of dissection; Guangqi Li, Zhongyi Duan, Shanshan Xie, Jingwei Yuan, Dehe Wang, Zebin Zhang, Xingzheng Li, Yajie Li, Chunning Mai, and Zhenfei Jiang for assistance with the sample collection; and Dr. Zhengsheng Xue for advice on the study. This manuscript has been released as a preprint at bioRxiv (Yan et al., 2018).

\section{SUPPLEMENTARY MATERIAL}

The Supplementary Material for this article can be found online at: https://www.frontiersin.org/articles/10.3389/fmicb. 2019.02126/full\#supplementary-material

Daber, R., Sukhadia, S., and Morrissette, J. J. (2013). Understanding the limitations of next generation sequencing informatics, an approach to clinical pipeline validation using artificial data sets. Cancer Genet. 206, 441-448. doi: 10.1016/ j.cancergen.2013.11.005

Dias, J., Marcondes, M. I., Motta, D. S. S., Cardoso, D. M. B., Noronha, M. F., Resende, R. T., et al. (2018). Bacterial community dynamics across the gastrointestinal tracts of dairy calves during preweaning development. Appl. Environ. Microbiol. 84, e2617-e2675. doi: 10.1128/AEM.02675-17

Dumonceaux, T. J., Hill, J. E., Hemmingsen, S. M., and Van Kessel, A. G. (2006). Characterization of intestinal microbiota and response to dietary virginiamycin supplementation in the broiler chicken. Appl. Environ. Microbiol. 72, 2815-2823. doi: 10.1128/aem.72.4.2815-2823.2006

Ericsson, A. C., Johnson, P. J., Lopes, M. A., Perry, S. C., and Lanter, H. R. (2016). A microbiological map of the healthy equine gastrointestinal tract. PLoS One 11:e166523. doi: 10.1371/journal.pone.0166523

Fanatico, A. C., Owens, C. M., and Emmert, J. L. (2009). Organic poultry production in the United States: broilers. J. Appl. Poult. Res. 18, 355-366. doi: $10.3382 / \mathrm{ps} /$ pey486

Fanatico, A. C., Pillai, P. B., Emmert, J. L., Gbur, E. E., Meullenet, J. F., and Owens, C. M. (2007a). Sensory attributes of slow-and fast-growing chicken genotypes raised indoors or with outdoor access. Poult. Sci. 86, 2441-2449. doi: 10.3382/ps.2007-00092

Fanatico, A. C., Pillai, P. B., Emmert, J. L., and Owens, C. M. (2007b). Meat quality of slow-and fast-growing chicken genotypes fed low-nutrient or standard diets and raised indoors or with outdoor access. Poult. Sci. 86, 2245-2255. doi: $10.1093 / \mathrm{ps} / 86.10 .2245$

Fanatico, A. C., Pillai, P. B., Hester, P. Y., Falcone, C., Mench, J. A., Owens, C. M., et al. (2008). Performance, livability, and carcass yield of slow-and fast-growing chicken genotypes fed low-nutrient or standard diets and raised indoors or with outdoor access. Poult. Sci. 87, 1012-1021. doi: 10.3382/ps.2006-00424

Gasaway, W. C., White, R. G., and Dan, F. H. (1976). Digestion of dry matter and absorption of water in the intestine and cecum of rock ptarmigan. Condor 78, 77-84. doi: 10.2307/1366918 
Gevers, D., Kugathasan, S., Denson, L. A., Vázquezbaeza, Y., Van, T. W., Ren, B., et al. (2014). The treatment-naive microbiome in new-onset Crohn's disease. Cell Host Microbe 15, 382-392. doi: 10.1016/j.chom.2014. 02.005

Gill, S. R., Pop, M., Deboy, R. T., Eckburg, P. B., Turnbaugh, P. J., Samuel, B. S., et al. (2006). Metagenomic analysis of the human distal gut microbiome. Science 312, 1355-1359.

Gong, J., Si, W., Forster, R., Huang, R., Yu, H., Yin, Y., et al. (2007). $16 \mathrm{~S}$ rRNA gene-based analysis of mucosa-associated bacterial community and phylogeny in the chicken gastrointestinal tracts: from crops to ceca. FEMS Microbiol. Ecol. 59, 147-157. doi: 10.1111/j.1574-6941.2006.00 193.x

Gu, S., Chen, D., Zhang, J. N., Lv, X., Wang, K., Duan, L. P., et al. (2013). Bacterial community mapping of the mouse gastrointestinal tract. PLoS One 8:e74957. doi: 10.1371/journal.pone.0074957

Johnson, T. J., Youmans, B. P., Noll, S., Cardona, C., Evans, N. P., Karnezos, T. P., et al. (2018). A consistent and predictable commercial broiler chicken bacterial microbiota in antibiotic-free production displays strong correlations with performance. Appl. Environ. Microbiol. 84, e318-e362. doi: 10.1128/AEM. 00362-18

Lavelle, A., Lennon, G., O’Sullivan, O., Docherty, N., Balfe, A., Maguire, A., et al. (2015). Spatial variation of the colonic microbiota in patients with ulcerative colitis and control volunteers. Gut 64, 1553-1561. doi: 10.1136/gutjnl-2014307873

Li, D., Chen, H., Mao, B., Yang, Q., Zhao, J., Gu, Z., et al. (2017). Microbial biogeography and core microbiota of the rat digestive tract. Sci. Rep. 8:45840. doi: $10.1038 /$ srep 45840

Lozupone, C., and Knight, R. (2005). UniFrac: a new phylogenetic method for comparing microbial communities. Appl. Environ. Microbiol. 71, 8228-8235. doi: 10.1128/aem.71.12.8228-8235.2005

Mabelebele, M., Alabi, O. J., Ngambi, J. W., Norris, D., and Ginindza, M. M. (2014). Comparison of gastrointestinal tracts and $\mathrm{pH}$ values of digestive organs of Ross 308 broiler and indigenous Venda chickens fed the same diet. Asian J. Anim. Vet. Adv. 9, 71-76. doi: 10.3923/ajava.2014.71.76

Magoč, T., and Salzberg, S. L. (2011). FLASH: fast length adjustment of short reads to improve genome assemblies. Bioinformatics 27, 2957-2963. doi: 10.1093/ bioinformatics/btr507

Matsui, H., Kato, Y., Chikaraishi, T., Moritani, M., Bantokuda, T., and Wakita, M. (2010). Microbial diversity in ostrich ceca as revealed by 16 S ribosomal RNA gene clone library and detection of novel Fibrobacter species. Anaerobe 16, 83-93. doi: 10.1016/j.anaerobe.2009.07.005

McCormack, U. M., Curiã, O. T., Buzoianu, S. G., Prieto, M. L., Ryan, T., Varley, P., et al. (2017). Exploring a possible link between the intestinal microbiota and feed efficiency in pigs. Appl. Environ. Microbiol. . 83, e317-e380. doi: 10.1128/ AEM.00380-17

Mead, G. C. (1989). Microbes of the avian cecum: types present and substrates utilized. J. Exp. Zool. 3, 48-54. doi: 10.1002/jez.1402520508

Nilakanta, H., Drews, K. L., Firrell, S., Foulkes, M. A., and Jablonski, K. A. (2014). A review of software for analyzing molecular sequences. BMC Res. Notes 7:830. doi: 10.1186/1756-0500-7-830

Oakley, B. B., Lillehoj, H. S., Kogut, M. H., Kim, W. K., Maurer, J. J., Pedroso, A., et al. (2014). The chicken gastrointestinal microbiome. FEMS Microbiol. Lett. 360, 100-112. doi: 10.1111/1574-6968.12608

Obst, B. S., and Diamond, J. M. (1989). Interspecific variation in sugar and amino acid transport by the avian cecum. J. Exp. Zool. Suppl. 252(Suppl. 3), 117-126. doi: 10.1002/jez.1402520519

Plummer, E., and Twin, J. (2015). A comparison of three bioinformatics pipelines for the analysis of preterm gut microbiota using $16 \mathrm{~S}$ rRNA gene sequencing data. J. Proteom. Bioinform. 8, 283-291.

Pollock, J., Glendinning, L., Wisedchanwet, T., and Watson, M. (2018). The madness of microbiome: attempting to find consensus "best practice" for $16 \mathrm{~S}$ microbiome studies. Appl. Environ. Microbiol. 84, e2617-e2627. doi: 10.1128/ AEM.02627-17

Quast, C., Pruesse, E., Yilmaz, P., Gerken, J., Schweer, T., Yarza, P., et al. (2013). The SILVA ribosomal RNA gene database project: improved data processing and web-based tools. Nucleic Acids Res. 41, D590-D596. doi: 10.1093/nar/gks1219

Rideout, J. R., He, Y., Navasmolina, J. A., Walters, W. A., Ursell, L. K., Gibbons, S. M., et al. (2014). Subsampled open-reference clustering creates consistent, comprehensive OTU definitions and scales to billions of sequences. PeerJ 2, e545. doi: 10.7717/peerj.545
Rizzo, J. M., and Buck, M. J. (2012). Key principles and clinical applications of "next-generation". DNA sequencing. Cancer Prev. Res. 5, 887-900. doi: 10.1158/ 1940-6207.capr-11-0432

Rosenbaum, M., Knight, R., and Leibel, R. L. (2015). The gut microbiota in human energy homeostasis and obesity. Trends Endocrinol. Metab. 26, 493-501. doi: 10.1016/j.tem.2015.07.002

Schmidt, C. J., Persia, M. E., Feierstein, E., Kingham, B., and Saylor, W. W. (2009). Comparison of a modern broiler line and a heritage line unselected since the 1950s. Poult. Sci. 88, 2610-2619. doi: 10.3382/ps.2009-00055

Sekelja, M., Rud, I., Knutsen, S. H., Denstadli, V., Westereng, B., Naes, T., et al. (2012). Abrupt temporal fluctuations in the chicken fecal microbiota are explained by its gastrointestinal origin. Appl. Environ. Microbiol. 78, 2941-2948. doi: 10.1128/AEM.05391-11

Sergeant, M. J., Constantinidou, C., Cogan, T. A., Bedford, M. R., Penn, C. W., and Pallen, M. J. (2014). Extensive microbial and functional diversity within the chicken cecal microbiome. PLoS One 9:e91941. doi: 10.1371/journal.pone. 0091941

Shaufi, M. A. M., Sieo, C. C., Chong, C. W., Gan, H. M., and Ho, Y. W. (2015). Deciphering chicken gut microbial dynamics based on high-throughput $16 \mathrm{~S}$ rRNA metagenomics analyses. Gut Pathogens 7:4. doi: 10.1186/s13099-0150051-7

Shin, N. R., Lee, J. C., Lee, H. Y., Kim, M. S., Whon, T. W., Lee, M. S., et al. (2014). An increase in the Akkermansia spp. population induced by metformin treatment improves glucose homeostasis in diet-induced obese mice. Gut 63, 727-735. doi: 10.1136/gutjnl-2012-303839

Smith, C. C., Snowberg, L. K., Gregory, C. J., Knight, R., and Bolnick, D. I. (2015). Dietary input of microbes and host genetic variation shape amongpopulation differences in stickleback gut microbiota. ISME J. 9, 2515-2526. doi: 10.1038/ismej.2015.64

Stanley, D., Geier, M. S., Chen, H., Hughes, R. J., and Moore, R. J. (2015). Comparison of fecal and cecal microbiotas reveals qualitative similarities but quantitative differences. BMC Microbiol. 15:51. doi: 10.1186/s12866-015-03886

Stanley, D., Geier, M. S., Hughes, R. J., Denman, S. E., and Moore, R. J. (2013). Highly variable microbiota development in the chicken gastrointestinal tract. PLoS One 8:e84290. doi: 10.1371/journal.pone.0084290

Stanley, D., Hughes, R. J., and Moore, R. J. (2014). Microbiota of the chicken gastrointestinal tract: influence on health, productivity and disease. Appl. Microbiol. Biotechnol. 98, 4301-4310. doi: 10.1007/s00253-014-5646-2

Stearns, J. C., Lynch, M. D. J., Senadheera, D. B., Tenenbaum, H. C., Goldberg, M. B., Cvitkovitch, D. G., et al. (2011). Bacterial biogeography of the human digestive tract. Sci. Rep. 1:170. doi: 10.1038/srep00170

Suzuki, T. A., and Nachman, M. W. (2016). Spatial heterogeneity of gut microbial composition along the gastrointestinal tract in natural populations of house mice. PLoS One 11:e163720. doi: 10.1371/journal.pone.0163720

Tillman, G. E., Haas, G. J., Wise, M. G., Oakley, B., Smith, M. A., and Siragusa, G. R. (2011). Chicken intestine microbiota following the administration of lupulone, a hop-based antimicrobial. FEMS Microbiol. Ecol. 77, 395-403. doi: 10.1111/j.1574-6941.2011.01119.x

Waite, D. W., and Taylor, M. W. (2014). Characterizing the avian gut microbiota: membership, driving influences, and potential function. Front. Microbiol. 5:223. doi: $10.3389 /$ fmicb.2014.00223

Wei, S., Morrison, M., and Yu, Z. (2013). Bacterial census of poultry intestinal microbiome. Poult. Sci. 92, 671-683. doi: 10.3382/ps.201202822

Wienemann, T., Schmitt-Wagner, D., Meuser, K., Segelbacher, G., Schink, B. Brune, A., et al. (2011). The bacterial microbiota in the ceca of Capercaillie (Tetrao urogallus) differs between wild and captive birds. Syst. Appl. Microbiol. 34, 542-551. doi: 10.1016/j.syapm.2011.06.003

Xiao, Y., Xiang, Y., Zhou, W., Chen, J., Li, K., and Yang, H. (2016). Microbial community mapping in intestinal tract of broiler chicken. Poult. Sci. 96, 1387-1393.

Yan, W., Sun, C., Yuan, J., and Yang, N. (2017). Gut metagenomic analysis reveals prominent roles of Lactobacillus and cecal microbiota in chicken feed efficiency. Sci. Rep. 7:45308. doi: 10.1038/srep45308

Yan, W., Zheng, J., Wen, C., Ji, C., Zhang, D., Chen, Y., et al. (2018). Efficacy of fecal sampling as a gut proxy in the study of chicken gut microbiota. bioRxiv 313577.

Yasuda, K., Oh, K., Ren, B., Tickle, T. L., Franzosa, E. A., Wachtman, L. M., et al. (2015). Biogeography of the intestinal mucosal and lumenal 
microbiome in the rhesus macaque. Cell Host Microbe 17, 385-391. doi: 10.1016/j.chom.2015.01.015

Yeoman, C. J., Chia, N., Jeraldo, P., Sipos, M., Goldenfeld, N. D., and White, B. A. (2012). The microbiome of the chicken gastrointestinal tract. Anim. Health. Res. Rev. 13, 89-99. doi: 10.1017/S1466252312000138

Yilmaz, P., Parfrey, L., Yarza, P., Gerken, J., Pruesse, E., Quast, C., et al. (2014). The SILVA and "All-species living tree project (LTP)" taxonomic frameworks. Nucleic Acids Res. 42, D643-D648. doi: 10.1093/nar/gkt1209

Zhao, L. L., Wang, G., Siegel, P., He, C., Wang, H. Z., Zhao, W. J., et al. (2013). Quantitative genetic background of the host influences gut microbiomes in chickens. Sci. Rep. 3:1163. doi: 10.1038/srep01163

Zhao, W., Wang, Y., Liu, S., Huang, J., Zhai, Z., He, C., et al. (2015). The dynamic distribution of porcine microbiota across different ages and gastrointestinal tract segments. PLoS One 10:e117441. doi: 10.1371/journal.pone.0117441
Conflict of Interest Statement: CJ, DZ, and YC were employed by the company Wen's Nanfang Poultry Breeding Co. Ltd.

The remaining authors declare that the research was conducted in the absence of any commercial or financial relationships that could be construed as a potential conflict of interest.

Copyright (c) 2019 Yan, Sun, Zheng, Wen, Ji, Zhang, Chen, Hou and Yang. This is an open-access article distributed under the terms of the Creative Commons Attribution License (CC BY). The use, distribution or reproduction in other forums is permitted, provided the original author(s) and the copyright owner(s) are credited and that the original publication in this journal is cited, in accordance with accepted academic practice. No use, distribution or reproduction is permitted which does not comply with these terms. 